\title{
Aspherical Supernovae and Oblique Shock Breakout
}

\author{
Niloufar Afsariardchi and Christopher D. Matzner \\ Department of Astronomy and Astrophysics, University of Toronto, \\ 50 St. George St, M5S 3H4, Toronto, Canada \\ email: afsariardchi@astro.utoronto.ca \\ email: matzner@astro.utoronto.ca
}

\begin{abstract}
In an aspherical supernova explosion, shock emergence is not simultaneous and nonradial flows develop near the stellar surface. Oblique shock breakouts tend to be easily developed in compact progenitors like stripped-envelop core collapse supernovae. According to Matzner et al. (2013), non-spherical explosions develop non-radial flows that alters the observable emission and radiation of a supernova explosion. These flows can limit ejecta speed, change the distribution of matter and heat of the ejecta, suppress the breakout flash, and most importantly engender collisions outside the star. We construct a global numerical FLASH hydrodynamic simulation in a two dimensional spherical coordinate, focusing on the non-relativistic, adiabatic limit in a polytropic envelope to see how these fundamental differences affect the early light curve of core-collapse SNe.
\end{abstract}

Keywords. supernovae: general, hydrodynamics, shock waves

\section{Introduction}

Shock breakout is the earliest observable phase of a supernova explosion and is associated with high energy radiation bursts in ultraviolet/X-ray frequencies and the release of high velocity ejecta (Klein \& Chevalier 1978). This radiation dominated shock accelerates while approaching the stellar surface due to steeply decreasing density profile of the SN progenitor and sets the ejecta into motion (e.g., Sakurai 1960, Matzner \& McKee 1999). The high energy photons diffuse out of the shock front when the optical depth of the progenitor $\tau$ drops below of the shock optical depth $\approx c / v_{s h}$, where $v_{s h}$ is the shock velocity. The breakout flash follows by envelope cooling emission from the deeper layers of the expanding ejecta in optical/near-UV bands.

Studying the early light curve and spectrum of core collapse supernovae enables us to obtain invaluable information about the nature and dynamics of the supernova shock and the characteristics of its progenitor (e.g., radius, envelope composition, and mass loss history) which cannot be inferred in later times when the light curve is governed by the radioactive decay. In recent years, the advancement of modern supernova surveys (e.g., Kaiser et al. 2002, Kim et al. 2016) along with the development of sensitive high energy detectors in UV, X-ray, and $\gamma$-ray frequencies has enhanced both the number of early detected supernovae and the accuracy of constraining supernova progenitor with $\mathrm{UV} / \mathrm{X}$-ray observations.

The early light curve of observed SNe are generally interpreted by spherical explosion models (Matzner \& McKee 1999, Nakar \& Sari 2010, Rabinak \& Waxman 2011), which have been extensively studied over past two decades. However, there has been growing evidence for aspherical scenarios when these models misinterpret the progenitor or cannot consistently explain the early lightcurve, spectrum, and polarization information. In the 
case of SN2011dh (Arcavi et al. 2011), for example, the progenitor was initially found to be a compact one based on the spherical models while it is now known as a yellow supergiant (YSG) from the stacked pre-SN images. This event also showed decreasing early polarization, suggesting aspherical structure in the fastest ejecta: (Mauerhan et al. 2015). For SN2008D, a Type Ib supernova (Soderberg et al. 2008), the polarization information and long $100 \mathrm{~s}$ detected X-ray flash supported aspherical breakout scenario (Couch et al. 2011).

Asymmetries seem theoretically inevitable in certain SN events. For instance, in the collapsar model of Gamma Ray Bursts (GRBs), the supernova signature is associated with the unbinding of the envelope by a highly asymmetric shock that is produced when a relativistic jet pierces a hole in the envelope (e.g., MacFayden \& Woosley 1999). Moreover, asymmetries can be inherent to the core collapse mechanism, whether in the jet-driven explosions (Piran et al. 2017), or neutrino-driven ones (Utrobin et al. 2017). Finally, aspherical dynamics also arise when the progenitor shape deviates from a perfect sphere; examples include rapidly rotating progenitor in which the progenitors are oblate as well as Roche lobe binary systems in which they are tidally deformed.

Here, we provide an overview on the current state of aspherical shock breakout and early light curve modelling and analysis and briefly comment on our latest numerical simulation and its results.

\section{Overview and Previous Work}

The effect of asphericity on the SN observables have been studied in previous numerical and analytical articles. A general review of the previous works is as follows:

Early Work. Suzuki \& Shigeyama (2010) investigate the effect aspherical energy deposition in a blue supergiant using a $2 \mathrm{D}$ hydrodynamical simulation. They derive the early light curve assuming a black-body radiation with a constant temperature along the progenitor. To treat the asymmetries, each patch of the progenitor is considered to radiate when the shock arrives and continue radiating for a constant amount of time. This has the drawback of ignoring the non-radial flows, which can completely change the shock emergence dynamics.

Jet-driven Hydrodynamic Simulations. The 2D hydrodynamic simulations of Couch et al. (2011) are targeted to reproducing the early light curve and the spectra of SN2008D. The explosion is invoked by jets that are active for about few seconds. The light curve and spectra modelling are done semi-analytically in post-processing. Unlike Suzuki \& Shigeyama (2010), they take into account the effect of non-radial flows by integrating black-body photon emission along thermalization front (i.e., where radiation and matter temperatures are equal). Their analysis results indicate that their reproduced light curve fits the observed light curve well, but their spectra poorly matched the observed one. As they point out, this can be due to simple black-body assumption that does not allow the photon color temperature to go above the thermalization temperature. Furthermore, the luminosity must have been set where the photons are able to diffuse upstream, not at thermalization front; this condition is met when dynamical time is equal to photon diffusion time, i.e., $t_{\mathrm{dyn}} \sim t_{\mathrm{diff}}$. The depth of thermalization front and the diffusion front may substantially differ, particularly in compact progenitors like the one considered in this study.

Oblique Shock Breakout. More recently, Matzner et al. (2013) analytically found that if the pattern speed of the shock progression along the stellar surface $v_{\phi}$ in aspherical explosions is about the shock speed $v_{s h}$, the shock dynamics are different from spherical case. Such "oblique shock breakouts" are predicted to suppress the direct breakout flash, limit the shock velocity, and cause non-radial flows to collide outside the star - the 

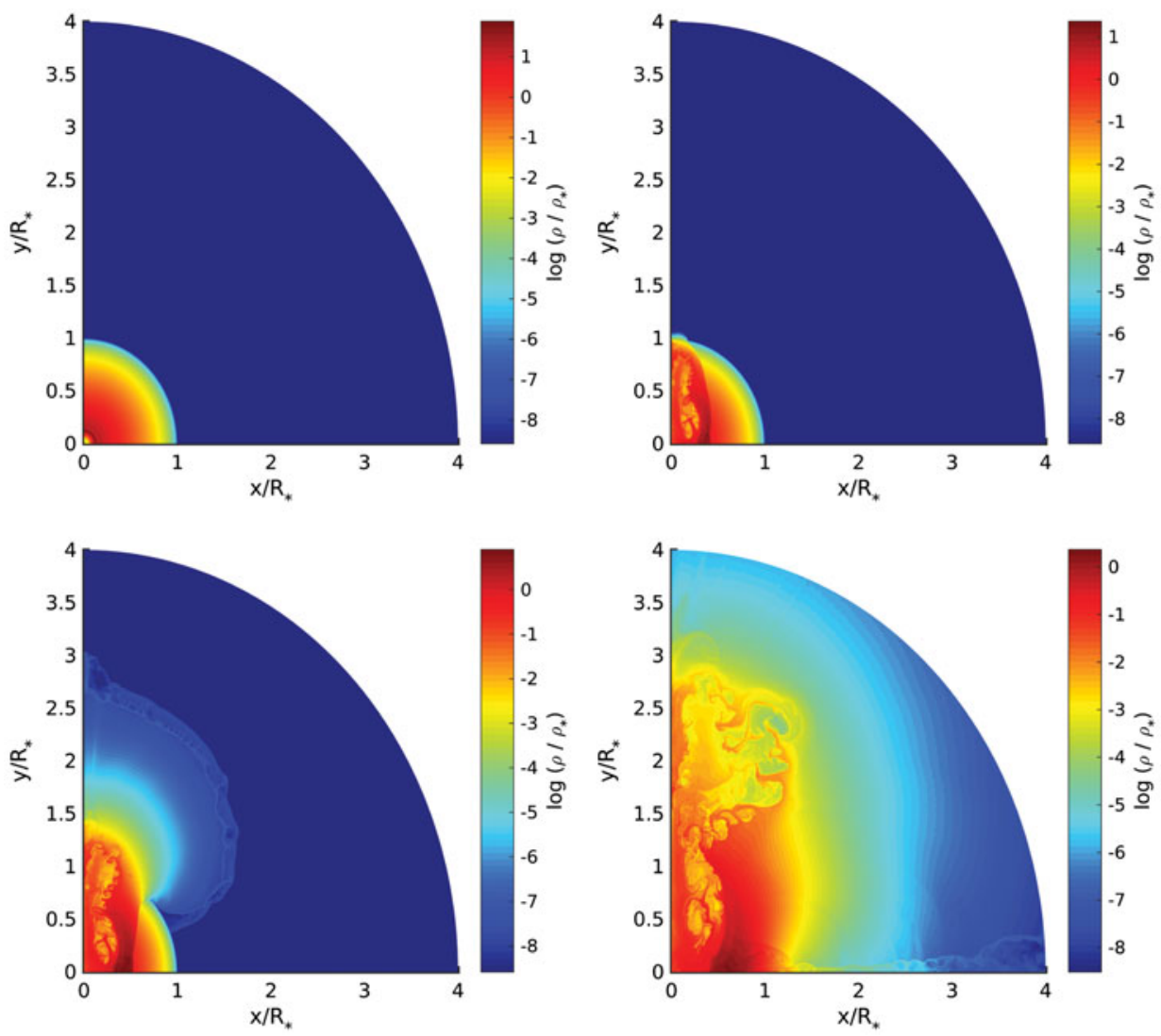

Figure 1. The snapshots of the density distribution of our scale-free simulations are shown. The top left panel shows the initial density distribution. In top right plot, the bipolar shock is emerged from the pole; the shock breaks normal to the stellar surface. The bottom left panel depicts the spray of the ejecta outside the stellar surface; at this stage the shock has become oblique. The bottom right panel marks the time when the shock has completely traversed the progenitor and the circumstellar collisions is happening along the equator.

predictions that are verified in the zoomed-in local simulations of Salbi et al. (2014). Despite providing the qualitative description of distinctive early light curve, these studies do not quantitatively model the early light curves or the spectrum.

Radiation Transfer Simulation. Recently, Suzuki et al. (2016) simulate aspherical explosion in a BSG progenitor using a single-group radiative transfer code and provide bolometric light curves for the simulation results. They, however, did not observe oblique shock breakout due to the insufficient degree of asphericity in their simulation.

\section{Simulation and Results}

We construct a global numerical FLASH (Fryxell 2000) hydrodynamic simulation in a two-dimensional uniform spherical-polar coordinate. The explosion is first triggered spherically in the center of a $n=3$ polytrope with energy deposited in few central cells where, resulting in a Sedov explosion with a sharp peak in the density and pressure profiles. The outcome of the spherical Sedov explosion is saved at time at early time when the shock is at about $0.2 R_{*}$. The saved velocity profile is then multiplied by $\cos ^{k}(2 \phi)$ to gain axisymmetrical momentum. A few density snapshots from our simulations are shown in 


\begin{tabular}{|c|c|c|c|c|c|}
\hline Model & $\mid M_{*}\left[2 \times 10^{33} \mathrm{~g}\right]$ & $R_{*}\left[6 \times 10^{10} \mathrm{~cm}\right]$ & $E_{*}\left[10^{51} \mathrm{erg}\right]$ & $\mathrm{X}$ & $\kappa\left[\mathrm{cm}^{2} / \mathrm{g}\right]$ \\
\hline RSG & 14 & 500 & 1 & 0.7 & 0.34 \\
\hline BSG & 15 & 49 & 1 & 0.7 & 0.34 \\
\hline Ic & 5 & 0.2 & 1 & 1 & 0.2 \\
\hline
\end{tabular}

Table 1. Simulations parameters that represent various explosions. The dynamical model is a complete $n=3$ polytrope.

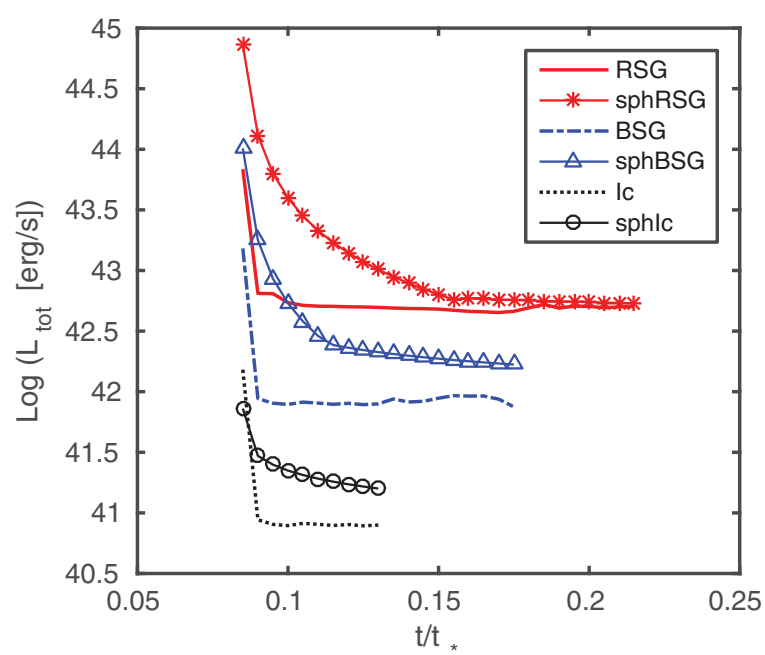

Figure 2. Angle-averaged apherical bolometric early light curve for three progenitors: RSG, BSG, and Type Ic model are shown. For comparison, the spherical early light curve of Nakar \& Sari (2010) is also shown for all three models. The time axis is normalized to the characteristic time $t_{*}=R_{*} \sqrt{M_{*} / E_{*}}$, where $R_{*}, M_{*}$, and $E_{*}$ are progenitor radius, progenitor mass, and explosion energy respectively shown in Table 1 .

Figure 1. In our simulations, we employ adiabatic flow and ignore gravity and relativity, rendering them scale free. However we post-process them to check these approximations (see Afsariardchi \& Matzner 2017), and to account or radiative transfer (absorptive emission and diffusion). In this process, we scale the simulations to three physical contexts: Red Supergiant (RSG), Blue supergiant (BSG), and compact Ic model, described in Table 1 . The extent of diffusion front, has revealed that our simulations are mainly relevant to compact progenitors and BSGs. For RSGs, the photon diffusion starts early on in the hydrodynamic evolution and thus our adiabatic assumption breaks. Moreover, we calculated the obliquity scales of our simulations based on their definition in Matzner et al. 2013 to see when and in which progenitors a normal shock turns to oblique shock.

In order to obtain bolometric luminosity light curves for each progenitor model, we find diffusion front and use diffusion approximation (details in Afsariardchi \& Matzner 2017) to calculate luminosity using a method similar to Chevalier (1992). In Figure 2, the early bolometric light curve of three different progenitors is shown for our aspherical simulation. For comparison, the spherical analytical models of Nakar \& Sari (2010) are also plotted. The aspherical light curves are all angle-averaged to show overall evolution of the energy release rate as oppose to observer-dependent light curves. As shown in Figure 2, the light curve of all three progenitors have a narrow peak in the early bolometric light curve. This peak is associated with shock breakout from the poles, when the shock is still normal 
to the stellar surface, but as the shock becomes oblique the luminosity significantly declines and the light curve enters a phase of constant luminosity. This is because the direct breakout flash is obscured under an optically thick spray of ejecta when the shock is oblique. Furthermore, comparing the constant part of aspherical light curves to the corresponding spherical ones, we find that the asherical light curves are few (i.e., $<3$ ) times less luminous than their spherical counterparts. This indicates that the oblique shock breakout emission is almost indistinguishable from the expanding ejecta phase of spherically symmetric SNe.

Deriving photon energies is more complicated; the assumption of thermalization that was made in prior works on aspherical explosion (e.g., in Suzuki \& Shigeyama 2010, Couch et al. 2011) does not necessarily hold as is the case for spherical explosion in compact progenitors (see e.g., Nakar \& Sari 2010). We find the thermalization front, color temperature, and band-dependent light curves in Afsariardchi \& Matzner (2017).

\section{References}

Afsariardchi, N. \& Matzner, D. C. 2017, in prep

Arcavi, I., Gal-Yam, A., Yaron, O. et al. 2011, ApJ, 742, L18

Chevalier, R. A. 1992, ApJ, 394, 599

Couch, S. M., Pooley, D., Wheeler, J. C., \& Milosavljević, M. 2016, ApJ, 727, 104

Fryxell, B. 2000, ApJS, 131, 273

Kaiser, N. \& Pan-STARRS Team 2002, Bull. of American Astro. Soc., 34, 1304

Kim, S.-L., Lee, C.-U., Park, et al. 2016, Journal of Korean Astronomical Society, 49, 37

Klein, R. I. \& Chevalier, R. A. 1978, ApJl, 223, L109

MacFadyen, A. I. \& Woosley, S. E. 1999, ApJ, 524, 262

Matzner, C. D. \& McKee, C. F. 1999, ApJ, 510, 379

Matzner, C. D., Levin, Y., \& Ro, S. 1999, ApJl, 779, 60

Mauerhan, J. C., Williams, G. G., Leonard, D. C., et al. 2015, MNRAS, 453, 4467

Nakar, E. \& Sari, R. 2010, ApJ, 725, 904

Piran, T., Nakar, E., Mazzali, P., \& Pian, E. 2017, ArXiv e-prints

Rabinak, I. \& Waxman, E. 2011, ApJ, 728, 63

Sakurai, A. 1960, Comm. Pure Appl. Math, 13, 353

Salbi, P., Matzner, C. D., Ro, S., \& Levin, Y. 2014, ApJ, 790, 71

Soderberg, A. M., Berger, E., Page, K. L., et al. 2008, Nature, 453, 469

Suzuki, A. \& Shigeyama, T. 2010, ApJl, 717, L154

Suzuki, A., Maeda, K., \& Shigeyama, T. 2016, ApJ, 825, 92

Utrobin, V. P., Wongwathanarat, A., Janka, H.-T., \& Mueller, E. 2017, ArXiv e-prints

\section{Discussion}

HiRschi: Do you also consider hydrogen poor progenitors?

AFSARIARDCHI: Yes, our type Ic model is hydrogen poor.

FRYER: Are your light curves sensitive to simulation resolution?

AfSARIARDCHI: We did a resolution study, and saw the same overall evolution, we, however, have not generated the light curve for our low resolution runs.

RAY: How does asphericity affect the relativistic flow?

AfSARIARDCHI: Asphericity put a new lower limit on the maximum speed of the flow. So, this new limit may prevent the ejecta to become relativistic. 